\title{
Stealing Death of Penaeus Vannamei and its Prevention and Control
}

\author{
Xiaojiang CHEN ${ }^{1}$, Jiabin WENG ${ }^{2}$, Quan WANG ${ }^{*}$ \\ ${ }^{1}$ Jiangsu Agri-animal Husbandry Vocational College. Jiangsu Taizhou, China \\ ${ }^{2}$ Jiangsu Hongyun Food Co., Ltd., Jiangsu Taizhou, China
}

*Corresponding Author: Quan WANG, Jiangsu Agri-animal Husbandry Vocational College. Jiangsu Taizhou, China

\begin{abstract}
In order to find out the causes of stealing death of Penaeus vannamei and find out the corresponding measures. Through behavior observation, external characteristics, microscopic examination and bacterial culture. The results showed that Vibrio parahaemolyticus was the pathogen of the disease, which grew rapidly and spread widely, and could be transmitted by shrimp seedlings or water sources. The outbreak of stealing death of Penaeus vannamei affected by its own disease resistance and environmental factors.
\end{abstract}

Keywords: Penaeus vannamei; Stealing death disease; Prevention and control; Vibrio parahaemolyticus

\section{INTRODUCTION}

In 2015, the output value of Penaeus vannamei exceeded 30 billion yuan in China. This variety has fast growth, relatively high meat yield and wide adaptability to salinity, which makes it develop rapidly in coastal and inland areas. However, in recent years, the morbidity of Penaeus vannamei is very high, and the morbidity time is long and the losses are heavy. Especially since 2010, the release of seedlings has started to get sick quickly, and the mortality rate is very high. In 2015, the incidence of cultured Penaeus vannamei in Baodi Dabai area of Tianjin was over 90\%. According to statistics, $15-17 \mathrm{hm}^{2}$ of shrimp ponds in Baodi have suffered from this disease after 30-40 days of seedling release, 10-14 $\mathrm{hm}^{2}$ of secondary seedlings have been released after all deaths, and the survival rate of the first batch of seedlings is $10 \%-20 \%$. The late culture period of Penaeus vannamei is a period of high disease incidence, with extremely low survival rate and increased culture risk. Basically, the shrimp seedlings started to get sick after being put into the shrimp pond for 15-20 days. Normal shrimps ate the disease and died shrimp, which caused the disease to spread rapidly and could not be controlled, resulting in heavy losses of Penaeus vannamei, mainly characterized by jejunum, empty stomach and stealing death. In this paper, the main symptoms, prevention and control of Penaeus vannamei were introduced for reference.

\section{Symptoms of STEALing DeATH OF PENAEUS VANNAMEI}

\subsection{Behavior Characteristics of Sick Shrimp}

Once the disease occurs in Penaeus vannamei, the shrimp will start to eat less or stop eating. Sick shrimps often float on the water surface or on the edge of aquaculture ponds, lacking vitality. It is often seen that some seriously ill shrimps jump on the water surface of aquaculture ponds and then sink vertically in a shock shape.

\subsection{Appearance and Morphology of Sick Shrimp}

By visual inspection, it can be found that there is no excess food in shrimp stomach and intestine, that is, empty stomach in jejunum. The body color is yellow, lighter and more transparent. Shrimp shells are not soft. Only at the late stage of disease, when shrimp has not eaten for a long time, and the body constitution of shrimp is getting better and better, shrimp shells will fade and soften gradually. The hepatopancreas is atrophied, its size is obviously abnormal, even some swelling, and its color is a little red (Figure 1). 


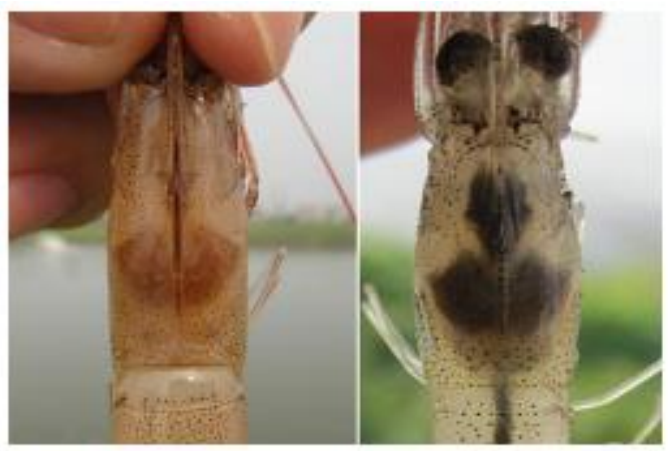

Fig1. Disease shrimp (left) and healthy shrimp (right)

\subsection{Microscopic Observation}

The pathogenesis of hepatopancreas can be divided into three stages: the first stage, the second stage, the second stage, the first stage, the second stage, the second stage, the second stage, the second stage, the third stage, the third stage, the third stage, the third stage, the third stage, the third stage, the third stage, the third stage, the third stage. In the middle stage, gourd-like atrophy increased, and there was no liquefied food in the hepatic duct. In the later stage, the cucurbit-like shape was severely atrophied, the cells responsible for absorbing nutrition almost did not exist, and the hepatic tubules were necrotic and even eroded. There are few oil droplets in the liver, and some even have no oil droplets (Figure 2). The stomach is red, and the walking and swimming feet of shrimps can be observed under the microscope (Figure 3), which indicates that the shrimps eat dead shrimps or become infected with sick shrimps, and the head and chest nails have white spots under the microscope (Figure 4), and there are many ciliates attached to the swimming feet (Figure 5).

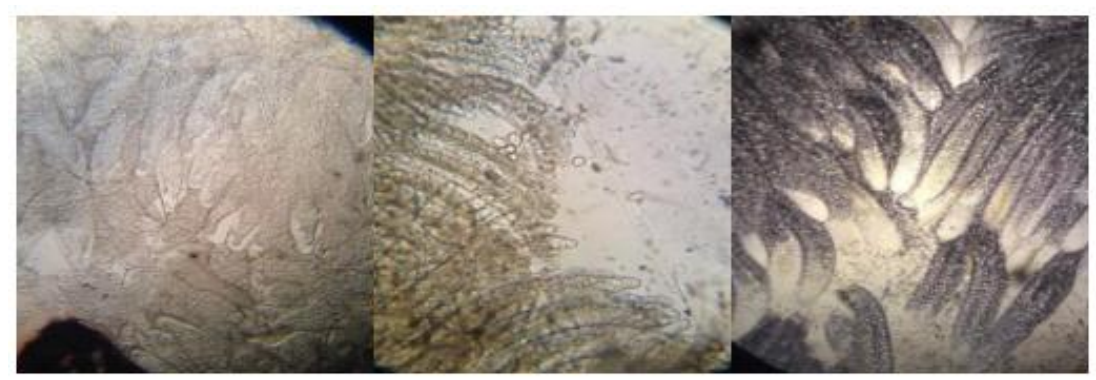

Figure2. Hepatopancreas

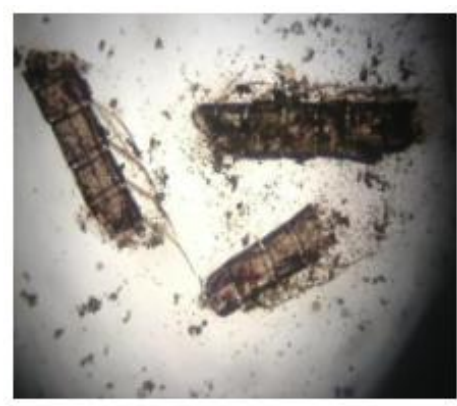

Figure3. Shrimp gastroscopy

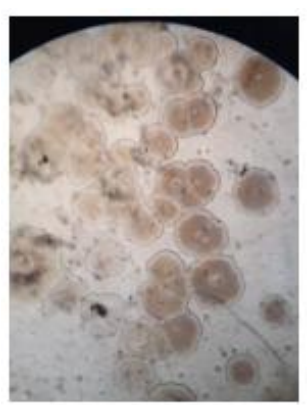

Figure4. Microscopic examination of the head and breast carapace of shrimp 


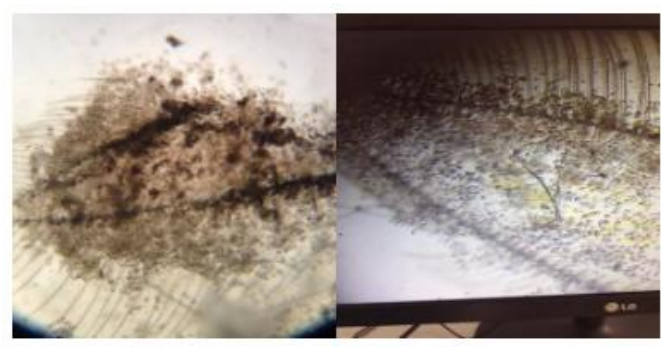

Figure5. Swimming feet attached to ciliates

\subsection{Selective Culture of Bacteria}

Bacteria were cultured in water, hepatopancreas and muscle with common medium and Vibrio selective medium, and a highly purified Vibrio strain could be isolated from hepatopancreas and muscle in bacteria selective medium (Figure 6).

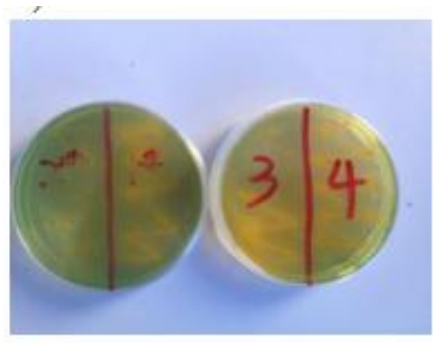

Figure6. Vibrio selective medium and normal medium

\section{Factors Causing Stealing Death of Penaeus Vannamei}

\subsection{Vibrio Parahemolyticus}

The main pathogenic factor affecting jejunal empty stomach in Penaeus vannamei is Vibrio parahaemolyticus [1]. This kind of bacteria is hydrochloric acid-eating bacteria, and it is difficult to propagate on the medium without salinity. It can multiply rapidly in saline water with a concentration of $30 \% \sim 60 \%$, with a cycle time of about eight to nine minutes, and it stops growing in the salinity environment lower than $0.5 \%$ or higher than $80 \%$. When $\mathrm{pH}$ is 8.5 , it is the most suitable environment for this bacterium, and it is also difficult to reproduce and grow below 6. However, this bacterium still has strong vitality, for example, it can survive for more than 1 month under suitable conditions and for more than 40 days in seawater. The distribution of Vibrio parahaemolyticus was detected in eastern China. The detection rate of Penaeus vannamei in coastal areas was $47.5 \%$ 66.5\%, the detection rate of fish and shrimp living in the sea was $45 \% \sim 48 \%$, and the detection rate was as high as $90 \%$ in summer [2].

\subsubsection{Transmission Route of Vibrio Parahaemolyticus in Culture}

Shrimp seedlings carry Vibrio parahaemolyticus: however, shrimp seedlings are transported from the nursery to the culture area and directly put into the culture pond without any disinfection and sterilization treatment, which becomes a major source of Vibrio parahaemolyticus infection.

There is Vibrio parahaemolyticus in the water source: the water source in the breeding area mainly comes from the side ditch, which is basically shared, without any means of treatment, and directly enters the water when necessary, and the quality of the water source is very poor, especially in the disease season, when the owner drains the water into the west. This is one of the reasons why contiguous farming areas are more prone to disease.

There are a large number of vibrios in the culture pond itself: in the old culture pond, the sludge that has been deposited for a long time can easily breed a large number of harmful bacteria, including Vibrio parahaemolyticus, which can multiply and infect explosively when the conditions are ripe.

\subsubsection{Harmful Mode of Vibrio Parahaemolyticus in Prawn Culture}

Vibrio parahaemolyticus, which entered the shrimp from various ways, caused confusion and imbalance in metabolism of Penaeus vannamei organism, which resulted in the decrease of enzyme activities in shrimp, and the shrimp could not normally carry out organism activities, resulting in death. On the other 
hand, Vibrio paralyticus infection destroyed the immune system of shrimp, and the immune function was damaged, which made the ability of disease prevention and disease resistance drop sharply, and further led to the disease and death of shrimp.

\subsection{Own Factors}

Parent problem, the development of aquaculture in China has promoted the expansion of the culture area of Penaeus vannamei, and the demand for shrimp seedlings has also increased. However, the development of parent shrimp culture technology is not standardized, and the management of breeding is imperfect, with many diseases, indiscriminate use of drugs and poor physical fitness, which has a great impact on the spawning and hatching of parent shrimp in the later stage, and the hatched shrimp seedlings are prone to deformity.

\subsection{Environmental Factors}

\subsubsection{Hydrogen Ion Concentration (PH)}

The adaptation range of Penaeus vannamei to $\mathrm{pH}$ is 7.5 9.5, 8.0 9.2 for juvenile shrimp and 7.5 8.8 for adult shrimp [3]. The high $\mathrm{pH}$ value increases the toxicity of ammonia water in water. The environment of high concentration ammonia water decreases the catalytic activity of enzymes and the stability of cell membrane in Penaeus vannamei, which affects the excretion system of shrimp and the balance of internal and external pressure to a certain extent. In addition, the gill tissue of prawn is easily eroded, which leads to the decrease of shrimp intake, the decline of body constitution and the slow growth, which leads to the long duration of shrimp poisoning death. The lower $\mathrm{pH}$ value leads to the lower $\mathrm{pH}$ value of blood in shrimp and the lower oxygen transport function in blood, which leads to difficulty in breathing. Therefore, too high or too low $\mathrm{pH}$ value will have adverse effects on shrimp, and if not treated in time, shrimp will be infected with germs due to weak constitution and abnormal immune system.

\subsubsection{Transparency and Water Color}

Transparency and water color can roughly reflect the quantity and species of plankton in water [4]. $25 \mathrm{~cm}$ to $40 \mathrm{~cm}$ is appropriate, and the transparency should be controlled between $40 \mathrm{~cm}$ and $60 \mathrm{~cm}$ in the middle and late period. The variety and number of plankton will affect the change of water color. Good colors should be yellow-brown and yellow-green, while abnormal colors such as light green, emerald green, strong green, white turbidity, sauce red, blue-green and old green should be avoided (Figure 710). There is a certain amount of floating swimming creatures in the pond, which will reduce the transparency, play the role of shading, reduce shrimp activities and reduce its energy consumption.

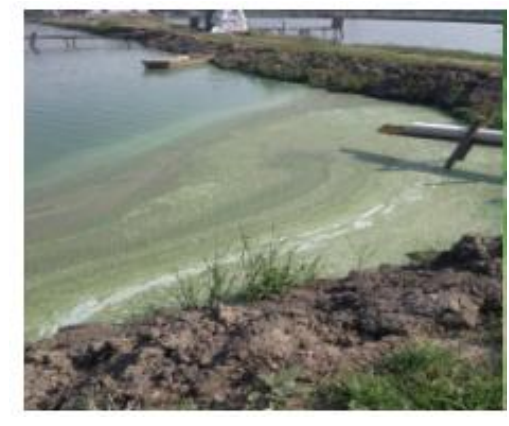

Figure7. Light green water color

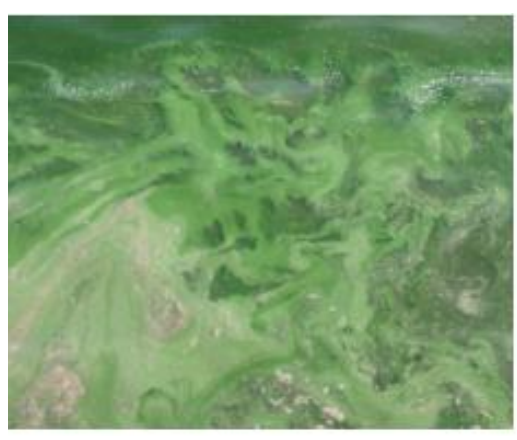

Figure8. Deep green watercolor 


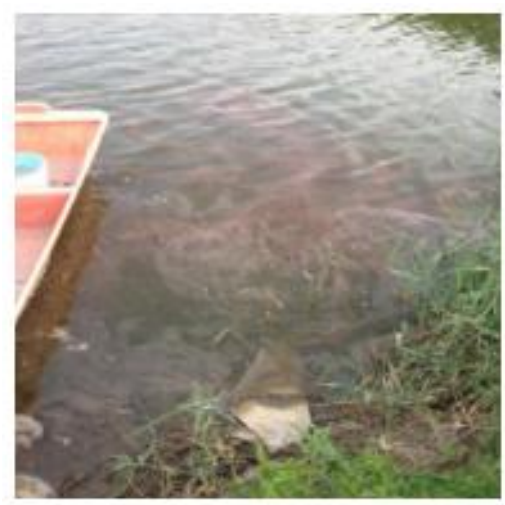

Figure9. Sauce red watercolor

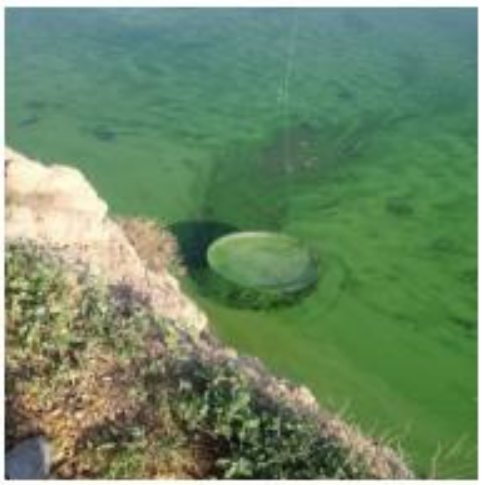

Figure10. Blue green watercolor

When moss is propagated in shrimp ponds, the height of moss can be as high as the depth of pond water. With the increase of temperature, moss will spread all over the pond, and when it is aging, the filamentous body will break off from the bottom of the pond and float on the water surface. Once the shrimp jumped onto the moss, the moss will entangle the shrimp like cotton wool, which can't break free, but can only dry up and die alive. In the high temperature season, moss turns yellow or white, and some even turn black, which seriously damages the bottom of the pond and gives off stench. There are many harmful algae in the water, and the dead algae and moss will produce harmful substances, which will lead to the poisoning of shrimp, and the decline of physique will lead to the death caused by Vibrio parahaemolyticus infection [5].

\subsubsection{Bottom Material}

In the process of aquaculture, many people attach great importance to disinfection and improvement of water bodies, rather than focusing on the bottom of ponds. The main living area of shrimp is at the bottom of the breeding pond. When the bottom of the pond deteriorates, due to the low dissolved oxygen, a large number of organic matters are difficult to decompose under anaerobic conditions, resulting in a large number of harmful substances, which reduces the resistance of prawns. When it reaches a certain level, it will cause a large-scale disease outbreak. At the same time, the feeding ability of shrimp will be weakened. If the amount of bait is constant, the residual bait will increase, and the residual bait will increase the pressure at the bottom, resulting in a vicious circle. Therefore, it is of great significance to supervise and improve the bottom quality (Figure 11).

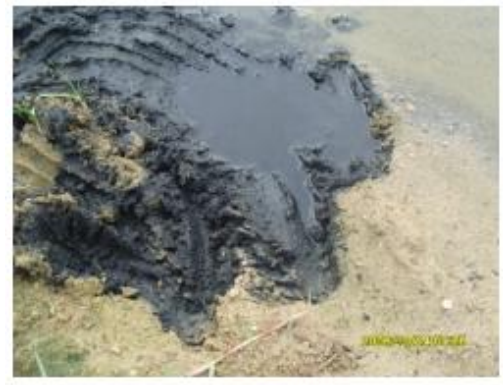

Figure11. Deteriorated bottom mud 


\subsubsection{Dissolved Oxygen}

Dissolved oxygen plays an important role in the success or failure of the whole process, and also affects the occurrence of diseases [6]. It is determined by many factors (such as weather, algae, aerator, etc.). The configuration of aerator is mainly to break the tension of water meter and make it dissolve more oxygen when the light intensity is uneven. The dissolved oxygen value in water is proportional to the conversion rate of water, $\mathrm{NH} 3$ and $\mathrm{NO} 2$ in water. In order to ensure high and stable yield, it is necessary to increase the dissolved oxygen content of water, especially the dissolved oxygen content at the bottom of pond. Lack of dissolved oxygen will easily reduce shrimp's intake of feed and lead to physical degradation.

\section{Pre-Control Method of Stealing Death of Penaeus Vannamei}

\subsection{Parent Shrimp}

Shrimp should be normal in body color and appearance, free of disease and damage, scientifically advanced in management, properly and regularly fed, so as to improve the shrimp's own physique and strengthen its ability to resist diseases, and while preventing diseases, it should minimize the use of drugs, especially antibiotics [7, 8]. In addition, when shrimp are docked from three transfer points, i.e., cultivation pool, temporary cultivation pool and indoor overwintering pool, they must be selected repeatedly and strictly checked [9].

\subsection{Shrimp Seedlings}

Shrimp seedlings should be produced routinely. Shrimp is strong, healthy and virus-free. Shrimp has high physical uniformity, transparent body color and strong vitality. Shrimp should have healthy tentacles and sharp open tail fan. The surface of shrimp should be clean and free from parasitic insects and damage. The stomach and intestines are full and orange-red; Shrimp can bounce flexibly and respond to external stimuli. In the static state, most shrimps are floating at a low level. After being stimulated by the water flow, they can flow at the top instead of drifting with the tide. In addition, to judge the quality of shrimp seedlings, several shrimps can be randomly placed in wet towels for 10 minutes, and then put into water. If the shrimps can survive, the shrimps tend to be qualified

\subsection{Regulation of $\mathrm{PH}$ Value}

If the $\mathrm{pH}$ value of the water is lower than 7.4, it can be increased by splashing appropriate amount of lime or other related drugs, or it can be selected to properly supplement the nutrition needed by algae growth, accelerate the culture and growth of phytoplankton, and consume too much $\mathrm{CO} 2$ in the water to increase the $\mathrm{pH}$ value of pond water. If the $\mathrm{PH}$ value of water is higher than 9.5, it can be adjusted by changing water or injecting new water or splashing some acidic substances (such as hydrochloric acid, glacial acetic acid, etc., the usage of which depends on the situation) to reduce the $\mathrm{pH}$ value of water.

\subsection{Transparency and Water Color}

Thoroughly disinfect aquaculture water before disinfecting seedlings, and cultivate fresh water quality by using organic fertilizer or low residual liquid fertilizer. The breeding of vibrio mainly needs to be in an organic environment. The water in shrimp ponds is thin, the content of organic suspended solids is low, and the growth of vibrio is slow and small, and the formation rate is high. Yellow and green is the best water color in aquaculture ponds, which is stable and difficult to turn into water. Maintain good water color, make a certain amount of phytoplankton in the pond, increase oxygen during the day, and reduce the influence of carbon dioxide, ammonia nitrogen and hydrogen sulfide prawns.

\subsection{Bottom Material}

The vertical distribution of vibrios in aquaculture water was detected by vibrio culture medium. The results showed that most vibrios were located at the bottom of pond [10] and attached to food, residues, organic debris and dead algae. Therefore, after the seedlings were released, the bottom was improved or cleaned every 5 days to about one week, and potassium persulfate disinfectant was sprinkled throughout the pond to kill the Vibrio in the water and bottom of the pond, and the number range was controlled at about 1000 strains $/ \mathrm{ml}$. 


\subsection{Dissolved Oxygen}

Generally, $3 \mathrm{~kW}$ aerator is installed in about $3 \mathrm{mu}$, so as to increase the aeration intensity and increase the average configuration of aerator. According to the analysis of aquaculture situation in Zhuhai in recent years, ponds with strong aerobic capacity have lower morbidity and higher yield. The development of all diseases is accompanied by the change of dissolved oxygen, low dissolved oxygen, rapid reproduction and rapid onset.

\subsection{Others}

Vibrio parahaemolyticus cannot survive in acidic environment. Therefore, the change of $\mathrm{pH}$ value should be controlled in shrimp ponds during the process of cultivation, and the environment with low threshold (usually in the range of 7.0-8.0) does not affect the normal growth of prawns. If the $\mathrm{pH}$ value of ponds changes, it can effectively delay the occurrence of shrimp diseases caused by Vibrio parahaemolyticus by using bacteria preparation or some suitable acid drugs to degrade the $\mathrm{pH}$ value of aquaculture water in time. During the shelling of shrimp shells, compound organic calcium and nutrients needed by shrimp bodies were splashed in the whole pond to supplement major elements and trace elements such as calcium, phosphorus, magnesium, potassium, etc. in water, which shortened the hard shell time after shelling, improved the physique of shrimp bodies and effectively prevented diseases [11].

The environment in which shrimp live is water, and the treatment factor is spread by water. It is difficult to cut off the source of infection. In addition, shrimp eat dead shrimp and sick shrimp, which leads to the rapid spread of shrimp disease. Once Penaeus vannamei suffers from jejunal empty stomach disease, there is basically no treatment. In the early stage, if it is found early, it can be taken orally (Longdan Xiegan Powder+Radix Isatidis $2 \mathrm{~g} / \mathrm{kg}$ with feed), and at the same time, some energy-supplementing drugs (glucose, brown sugar) and some disinfection drugs (dibromohydantoin, Bdellovibrio bacteriovorus $500 \mathrm{ml} / \mathrm{mu}$ ) must be sprinkled. However, once the disease of stealing death from jejunum and stomach occurs in shrimp, it is difficult to treat it thoroughly, and good prevention and cure is the key to control the disease.

\section{SUMMARY}

Penaeus vannamei culture is a high-risk and high-return investment. In order to obtain higher economic benefits, we must follow scientific farming methods and know the conventional prevention and control skills of shrimp diseases. Nowadays, the environmental conditions and technology of shrimp farming are becoming more and more complex. In recent years, shrimp diseases appear more and more frequently, and once they occur, they cannot be treated. The mortality rate can reach $70 \%-80 \%$ in $4-5$ days. Therefore, at the beginning of aquaculture, prevention and control work must be started, water quality should be regularly detected (such as algae, $\mathrm{pH}$, ammonia nitrogen, nitrite, $\mathrm{DO}$ ), and adjustment, bottom modification, disinfection and detoxification should be carried out according to changes in water environment, so that shrimp can live in a suitable environment

\section{ACKNOWLEDGMENT}

This research was financially supported by "Qing Lan Project of Jiangsu Province" ( 〔2018] 12)and "311 Talents Project in Taizhou City" (2017Ш-804), College Students' Innovative Entrepreneurial Training Plan Program (202012806019Y).

\section{REFERENCES}

[1] Zhai Xiumei, Wang Bin, Mao Lianju. Effects of Vibrio parahaemolyticus on physiological and biochemical indexes of Penaeus vannamei [J]. Journal of Shanghai Fisheries University, 2007(2); 16-18

[2] Ye xiaojing. study on immunity of Vibrio vaccine of prawn [J]. journal of marine fisheries research. 1990.(32); 13-18

[3] Shen Wenying, Hu Hongguo, Pan Yajuan. Effects of temperature and $\mathrm{pH}$ value on digestive enzyme activity of Penaeus vannamei [J]. oceanologia et limnologia sinica.2004.35 (6): 543-548.

[4] Cao Yan, Pond Water Color and Regulation Measures [J]. Heilongjiang Aquatic Products, [2] Chen Rong, Ma Guihua. Pond Algae and Management [J]. Jiangxi Aquatic Science and Technology, 2011(1); 44-46.

[5] Sun Guoming, Tang Jianhua, et al. Toxicity of ammonia nitrogen and nitrite to Penaeus vannamei [J]. Aquaculture, 2002:(2),22-24.

[6] Zhu Mou. Study on the influencing factors of dissolved oxygen in water [J]. China Agricultural Press, 2010(1); 30-35. 
[7] Wang Jiqiao. Healthy culture technology of Penaeus vannamei: I Biology of Penaeus vannamei [J]. Fisheries Science, 2002,21(5):43-46.

[8] Shen Wenying, Yang Huijun. Research status of disease and control of Penaeus vannamei [J]. Water Resources and Fisheries, 200424(1); 58-60

[9] Liu Jianyong. Cultivation technology of high-quality seedlings of Penaeus vannamei [J]. Guangdong Ocean University, 201401(2); 81-83

[10] Zhao Zeng-yuan, Li Tian-Bao, Guo Wen, et al. Study on the influence of bottom sludge on the water environment of prawn pond and the method of bottom improvement [J]. Marine Science, 1994(3):5-8.

[11] Zhai Xiumei, Wang Bin, Mao Lianju, Guo Yu, etc., Effects of Vibrio parahaemolyticus on Physiological and Biochemical Indexes of Penaeus vannamei, Journal of Shanghai Fisheries University, Vol. 16, No.2, 2007.3

Citation: Xiaojiang CHEN, Jiabin WENG, Quan WANG, "Stealing Death of Penaeus Vannamei and its Prevention and Control”, International Journal of Innovative Studies in Aquatic Biology and Fisheries, 6(4), pp. 13-20. DOI: https:// doi.org/10.20431/2454-7670.0604003

Copyright: (C) 2020 Authors, this is an open-access article distributed under the terms of the Creative Commons Attribution License, which permits unrestricted use, distribution, and reproduction in any medium, provided the original author and source are credited. 